University of Thi-Qar Journal Vol.10 No.4 Dec 2015

Web Site: https://jutq.utq.edu.iq/index.php/main Email: journal@jutq.utq.edu.iq

\title{
Preparation and Charactrization of 1,3- dipolar cycloaddition of nitrones with but-2-ynedioic acid
}

\author{
https://doi.org/10.32792/utq/utj/vol10/4/4
}

\author{
Raad.J.Ali \\ Directorate-General for Education in Dhi Qar \\ (Department of Nasiriyah)
}

\section{Abstract:}

Some nitrones(1-3), derived from N-p-tolylhydroxylamine with substituted benzaldehyde such as(4-Chlorobenzaldehyde,4-Florobenzaldehyde, 4-Nitrobenzaldehyde ) .Ther reaction with but-2-ynedioic acid include 1,3- dipolar cycloaddition reaction to give isoxazoles (4-6), They have been identified by ${ }^{1} \mathrm{HNMR}$, IR and Mass spectra

keyword: nitrones, isoxazole, 1,3- dipolar cycloaddition, but-2-ynedioic acid

\section{Introduction:}

The reactions of nitrones dipoles play an important role in the history of cycloaddition reaction . The 1,3- dipolar cycloaddition also known as the Huisgen cycloaddition ${ }^{1}$ is a classic reaction in organic chemistry consisting of the reaction of dipolarophile with a 1,3- dipolar compound ${ }^{2}$ that allows the production of various five -membered heterocycles ${ }^{3}$. High specificity stereoselectivity associated with these reactions make them synthetically important ${ }^{4-7}$.It has been found that 1,3 - dipolar cycloaddition reaction proceed through a concerted mechanism ${ }^{8}$.Most of dipolarophile are alkenes ${ }^{9,10}$, alkynes ${ }^{11}$ and molecules possessing related hetero atom functional groups (such as carbonyls ${ }^{12-14}$ and nitriles ${ }^{15-18}$ ).Both inter and intra molecular nitrone and alkynes cycloaddition reaction have received attention of heterocycles of biological interest ${ }^{19-22}$.

\section{Experimental}

\subsection{Apparatuses:}

Melting points were determind using a Gallenkamp melting point apparatus .

Proton NMR spectra were recorded on a Bruker DRX 400 Advance spectrometer at 500 $\mathrm{MHz}$ and $125 \mathrm{MHz}$,respectively,using deuterated solvents and TMS as an internal standard Chemical shifts are reported as $\delta$ values in ppm. Infrared spectra were obtained 
by FT-IR-1600 Perkin-Elmer spectrophotometer. Thin layer chromatography (TLC) was performed on aluminum sheets silica gel from merk.Column chromatography was carried out using Merck silica gel (230-400 mesh). The TLC spots were visualized in UV and $\mathrm{I}_{2}$. Mass spectra recorded on High-resolution mass spectra were recorded on an ESI-TOF Mariner Spectrometer (Perspective Biosystem)

\subsection{Preparation Methods}

\subsubsection{Preparation of the nitrones(1-3)}

The N-p-tolylhydroxylamine was prepared from nitro toluene according to $\operatorname{ref}^{26}$ and $\alpha$-aryl-N-phenyl nitrones (A1-3) from the substituted benzaldehyde and $\mathrm{N}$-p-tolylhydroxylamine according to $\operatorname{ref}^{27-34}$.

\subsubsection{Preparation of the isoxazoles(4-6)}

To A stirred solution of the nitrones (1-3) (5 mmole)[1.28 mg from comp.(1) and 1.145 $\mathrm{mg}$ from comp.(2) and $1.225 \mathrm{mg}$ from comp.(3) ] in dry toluene $(50 \mathrm{ml})$ was added to but-2-ynedioic acid ( 5 mmole) $[0.57 \mathrm{mg}]$ and the solution was heated at refluxed for (48$72 \mathrm{~h}$ ). The resulting mixture was evaporated under reduced pressure.

The crude product was purified by column chromatography on silica gel eluting to give pure isoxazoles (4-6).

\subsubsection{3-(4-nitrorophenyl)-2-p-tolyl-2,3-dihydroisoxazole-4,5-dicarboxylic acid(4)}

The product (4) was isolated by column chromatography on silica gel eluting with benzene $\backslash$ methanol(8:2) as a brown solid product in $60 \%$ yield, $\mathrm{m} . \mathrm{p}=254-255 \mathrm{C}^{\circ}$.

IR: $1751 \mathrm{~cm}^{-1}(\mathrm{C}=\mathrm{O}), 3300 \mathrm{~cm}^{-1} \mathrm{~s}(\mathrm{OH}), 1455 \mathrm{~cm}^{-1}(\mathrm{C}=\mathrm{C}), 1300 \mathrm{~cm}^{-1} \mathrm{w}(\mathrm{C}-\mathrm{N}) ; 1023 \mathrm{~cm}$ ${ }^{-1} \mathrm{~m}\left(\mathrm{NO}_{2}\right.$ Asym), $1340 \mathrm{~cm}^{-1} \mathrm{w}\left(\mathrm{NO}_{2}, \mathrm{sym}\right)$;

${ }^{1} \mathrm{HNMR}: \delta 11.58 \mathrm{ppm}(\mathrm{s}, 2 \mathrm{H})$ Carboxylic Acid O-H, 7.10- $8.52 \mathrm{ppm}(\mathrm{m}, 8 \mathrm{H}$ aromatic), $5.46 \mathrm{ppm}(\mathrm{s}, 1 \mathrm{H}), 2.65 \mathrm{ppm}$ (s,3H) Fig. (2 ); Mass: $\mathrm{mlz}=370[\mathrm{M}]^{+}$.Fig.(5)

\subsubsection{3-(4-fluorophenyl)-2-p-tolyl-2,3-dihydroisoxazole-4,5-dicarboxylic acid (5):}

The product (5) was isolated by column chromatography on silica gel eluting with benzenel methanol(8:2) as a brown solid product in $51 \%$ yield ,m.p $=186 \mathrm{C}^{\circ}$.

IR: $1747(\mathrm{C}=\mathrm{O}), 3350 \mathrm{br}(\mathrm{OH}), 1423(\mathrm{C}=\mathrm{C}), 1307 \mathrm{w}(\mathrm{C}-\mathrm{N})$;

${ }^{1} \mathrm{HNMR}: \delta 11.55 \mathrm{ppm}(\mathrm{s}, 2 \mathrm{H})$ Carboxylic Acid O-H, 6.91- $7.54 \mathrm{ppm}$ (m,8 $\mathrm{H}$ aromatic), $5.66 \mathrm{ppm}(\mathrm{s}, 1 \mathrm{H}), 2.51 \mathrm{ppm}(\mathrm{s}, 3 \mathrm{H})$ Fig.(3 ); Mass: $\mathrm{mlz}=343[\mathrm{M}]^{+}$. .Fig. ( 6 )

\subsubsection{3-(4-chlorophenyl)-2-p-tolyl-2,3-dihydroisoxazole-4,5-dicarboxylic acid (6)} the product (6) was isolated by column chromatography on silica gel eluting with benzenel methanol(8:2) as a yellow solid product in $55 \%$ yield , m. $\mathrm{p}=210-212 \mathrm{C}^{\circ}$. IR: $1747 \mathrm{~cm}^{-1}(\mathrm{C}=\mathrm{O}), 3330 \mathrm{~cm}^{-1}$ br $(\mathrm{OH}), 1492 \mathrm{~cm}^{-1}(\mathrm{C}=\mathrm{C}), 1311 \mathrm{~cm}^{-1} \mathrm{~m}(\mathrm{C}-\mathrm{N})$, ${ }^{1}$ HNMR : $\delta 11.58$ ppm (s,2H) Carboxylic Acid O-H, 7.17- 7.59 ppm (m,8 H aromatic), 5.46 ppm (s,1H), 2.65 ppm (s,3H) Fig.(4); Mass: $\mathrm{mlz}=359$ [M] ${ }^{+}$. Fig.(7) 
Web Site: https://jutq.utq.edu.iq/index.php/main Email: journal@jutq.utq.edu.iq

\section{Results and Discussion}

The nitrones(1-3) used in this study were prepared from the corresponding aldehyde with $\mathrm{N}$-p-tolylhydroxylamine ${ }^{23,24}$ Equation(1)

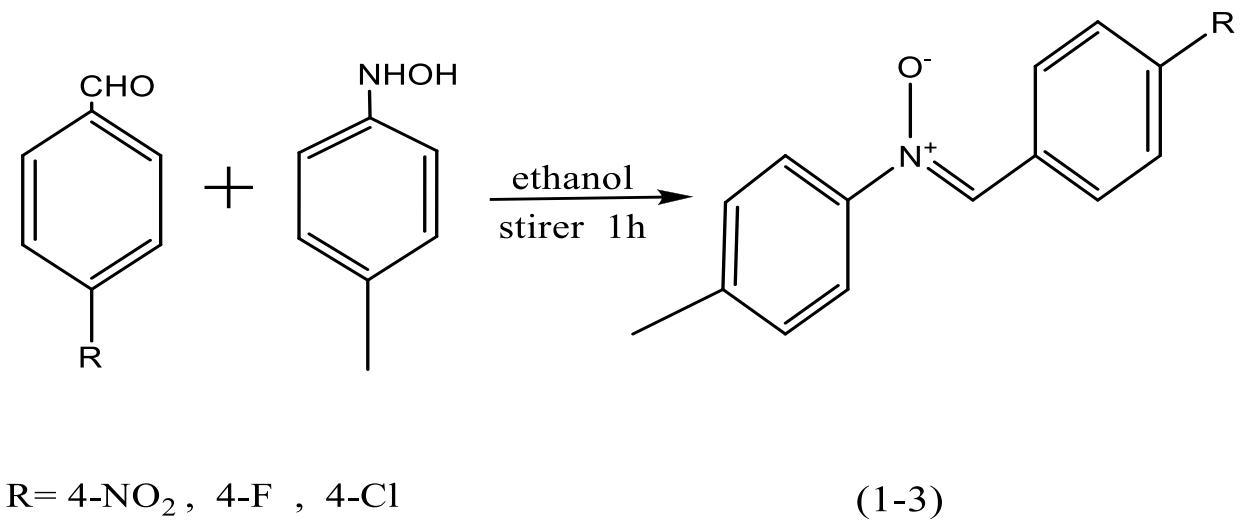

Equation(1)

Preparation of compound(4-6)

The cycloaddition of nitrones (1-3) with but-2-ynedioic acid were carried out by refluxing (48-72 h) in dry toluene at $110{ }^{\circ} \mathrm{C}$ to give isoxazoles(4-6). In all cases, The compounds were purified by column chromatography ${ }^{25}$ allowed the isolation of pure compounds. Equation(2)

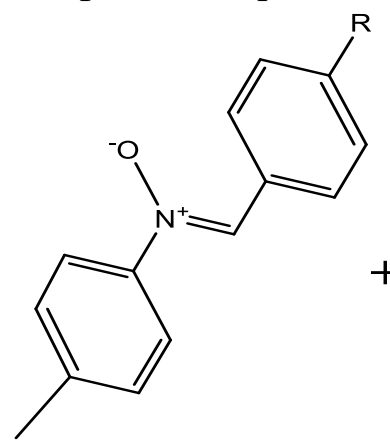

$(1-3)$

$$
\mathrm{R}=4-\mathrm{NO}_{2}, 4-\mathrm{F} \quad, 4-\mathrm{Cl}
$$

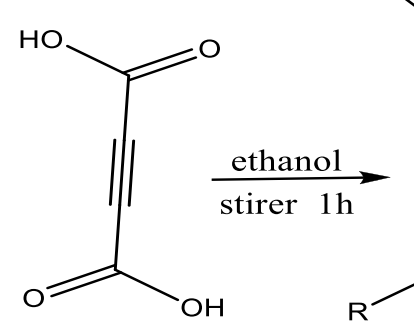<smiles>Cc1ccc(N2OC(C(=O)O)=C(C(=O)O)C2c2ccc(F)cc2)cc1</smiles>

$(4-6)$

Equation(2)

The obtained isoxazoles were characterized spectroscopically. The formation of the cycloadducts was established by the FTIR, ${ }^{1}$ HNMR and Mass spectroscopy .The ${ }^{1}$ HNMR spectrum of general structure isoxazoles (4-6)in Fig(1) list in table(1) spectrum showed a singlet at $\delta 2.51-2.65 \mathrm{ppm}$ for $\left(\mathrm{C}^{-} \mathrm{CH}_{3}\right)$., a multiplet at $\delta 6.91-8.52 \mathrm{ppm}$ for the 
University of Thi-Qar Journal Vol.10 No.4 Dec 2015

Web Site: https://jutq.utq.edu.iq/index.php/main Email: journal@jutq.utq.edu.iq

aromatic protons, a singlet at $\delta 5.46-5.66 \mathrm{ppm}$ for proton(a) and a singlet at $\delta 11.55-11.58$ ppm for acid proton,Fig.(2-4)

The IR spectrum included a peak at $3059-3150 \mathrm{~cm}^{-1}$ for the $\mathrm{NH}_{2}$ stretch and the sharp peak at $1647-1651 \mathrm{~cm}^{-1}$ for $\mathrm{C}=\mathrm{O}$ stretch

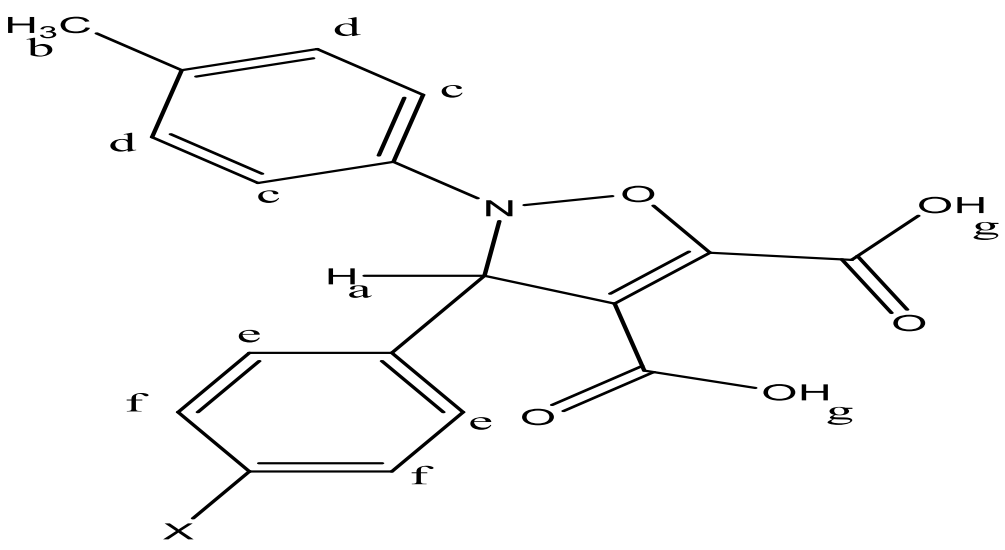

Fig(1) general structure of isoxazoles

Table (1): ${ }^{1}$ HNMR spectral data (ppm) for isoxazoles(4-6)

\begin{tabular}{|c|c|c|c|c|c|c|c|c|}
\hline Comp. & $\mathrm{X}$ & $\mathrm{H}_{\mathrm{a}}$ & $\mathrm{H}_{\mathrm{b}}$ & $\mathrm{H}_{\mathrm{c}}$ & $\mathrm{H}_{\mathrm{d}}$ & $\mathrm{H}_{\mathrm{e}}$ & $\mathrm{H}_{\mathrm{f}}$ & $\mathrm{H}_{\mathrm{g}}$ \\
\hline 4 & $4-\mathrm{NO}_{2}$ & $\begin{array}{c}5.47 \\
(\mathrm{~s}, 1 \mathrm{H})\end{array}$ & $\begin{array}{c}2.65 \\
(\mathrm{~s}, 3 \mathrm{H})\end{array}$ & $\begin{array}{c}7.1 \\
(\mathrm{~d}, 2 \mathrm{H})\end{array}$ & $\begin{array}{c}7.56 \\
(\mathrm{~d}, 2 \mathrm{H})\end{array}$ & $\begin{array}{c}8.27 \\
(\mathrm{~d}, 2 \mathrm{H})\end{array}$ & $\begin{array}{c}8.52 \\
(\mathrm{~d}, 2 \mathrm{H})\end{array}$ & $\begin{array}{c}11.58 \\
(\mathrm{~s}, 2 \mathrm{H})\end{array}$ \\
\hline 5 & $4-\mathrm{F}$ & $\begin{array}{c}5.66 \\
(\mathrm{~s}, 1 \mathrm{H})\end{array}$ & $\begin{array}{c}2.51 \\
(\mathrm{~s}, 3 \mathrm{H})\end{array}$ & $\begin{array}{c}6.91 \\
(\mathrm{~d}, 2 \mathrm{H})\end{array}$ & $\begin{array}{c}7.23 \\
(\mathrm{~d}, 2 \mathrm{H})\end{array}$ & $\begin{array}{c}7.32 \\
(\mathrm{~d}, 2 \mathrm{H})\end{array}$ & $\begin{array}{c}7.5 \\
(\mathrm{~d}, 2 \mathrm{H})\end{array}$ & $\begin{array}{c}11.55 \\
(\mathrm{~s}, 2 \mathrm{H})\end{array}$ \\
\hline 6 & $4-\mathrm{Cl}$ & 5.46 & 2.65 & $7.17-$ & 7.45 & 7.57 & 7.59 & 11.58 \\
& & $(\mathrm{~s}, 1 \mathrm{H})$ & $(\mathrm{s}, 3 \mathrm{H})$ & $7.21(\mathrm{~d}, 2 \mathrm{H})$ & $(\mathrm{d}, 2 \mathrm{H})$ & $(\mathrm{d}, 2 \mathrm{H})$ & $(\mathrm{d}, 2 \mathrm{H})$ & $(\mathrm{s}, 2 \mathrm{H})$ \\
& & & & & & & \\
\hline
\end{tabular}


University of Thi-Qar Journal Vol.10 No.4 Dec 2015

Web Site: https://jutq.utq.edu.iq/index.php/main Email: journal@jutq.utq.edu.iq

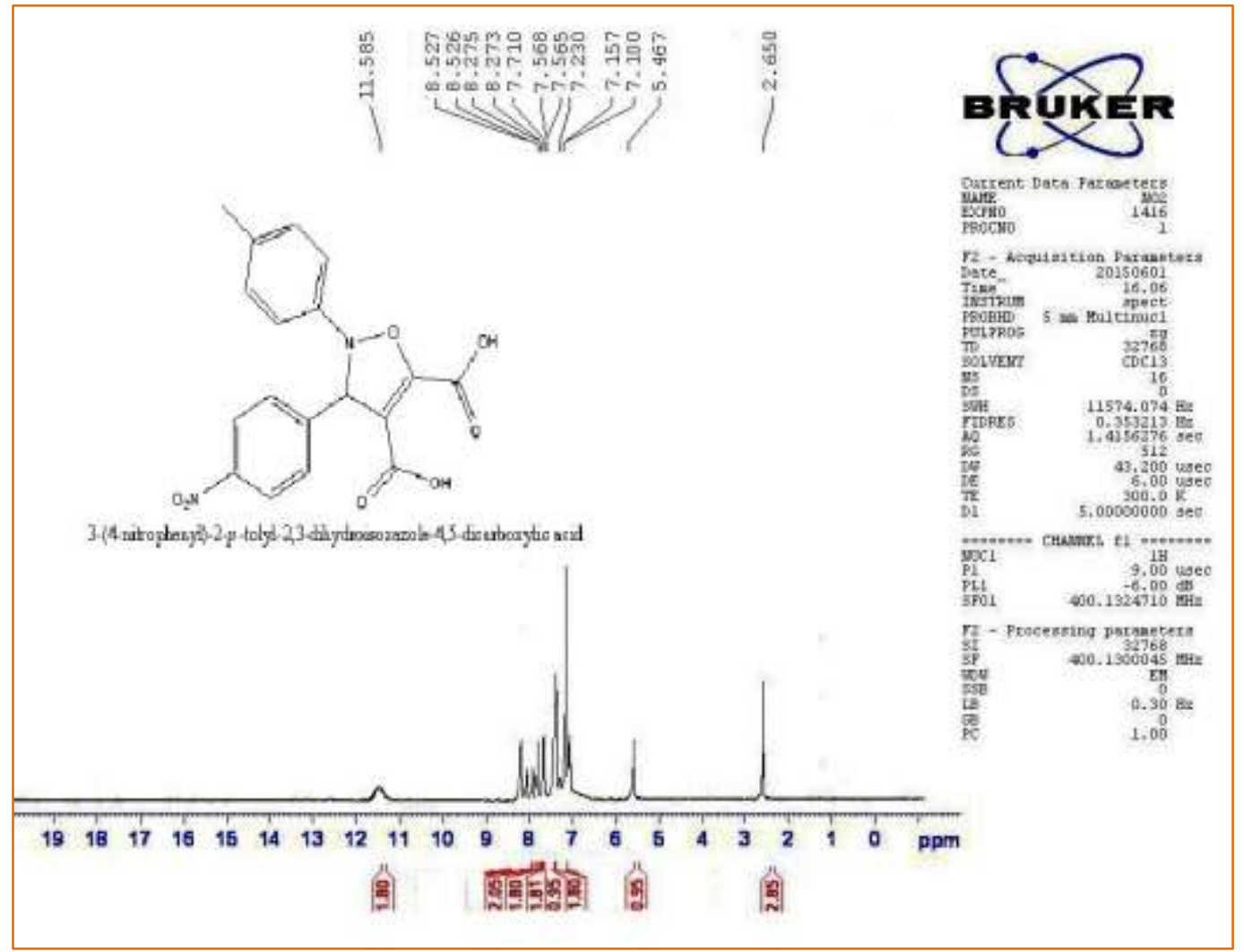

Fig (2) ${ }^{1}$ HNMR for compound (4)

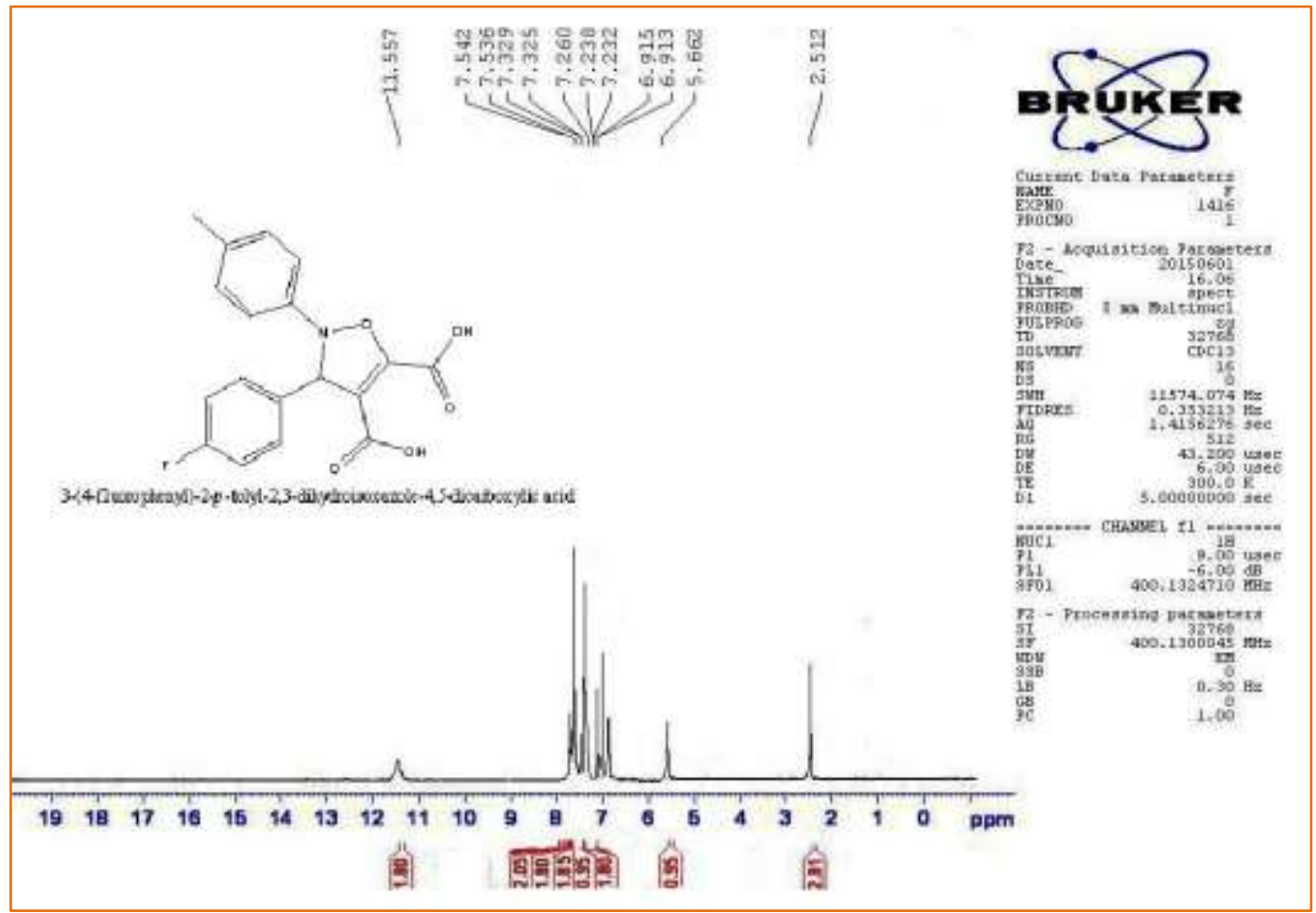

Fig (3) ${ }^{1}$ HNMR for compound (5) 
University of Thi-Qar Journal Vol.10 No.4 Dec 2015

Web Site: https://jutq.utq.edu.iq/index.php/main Email: journal@jutq.utq.edu.iq

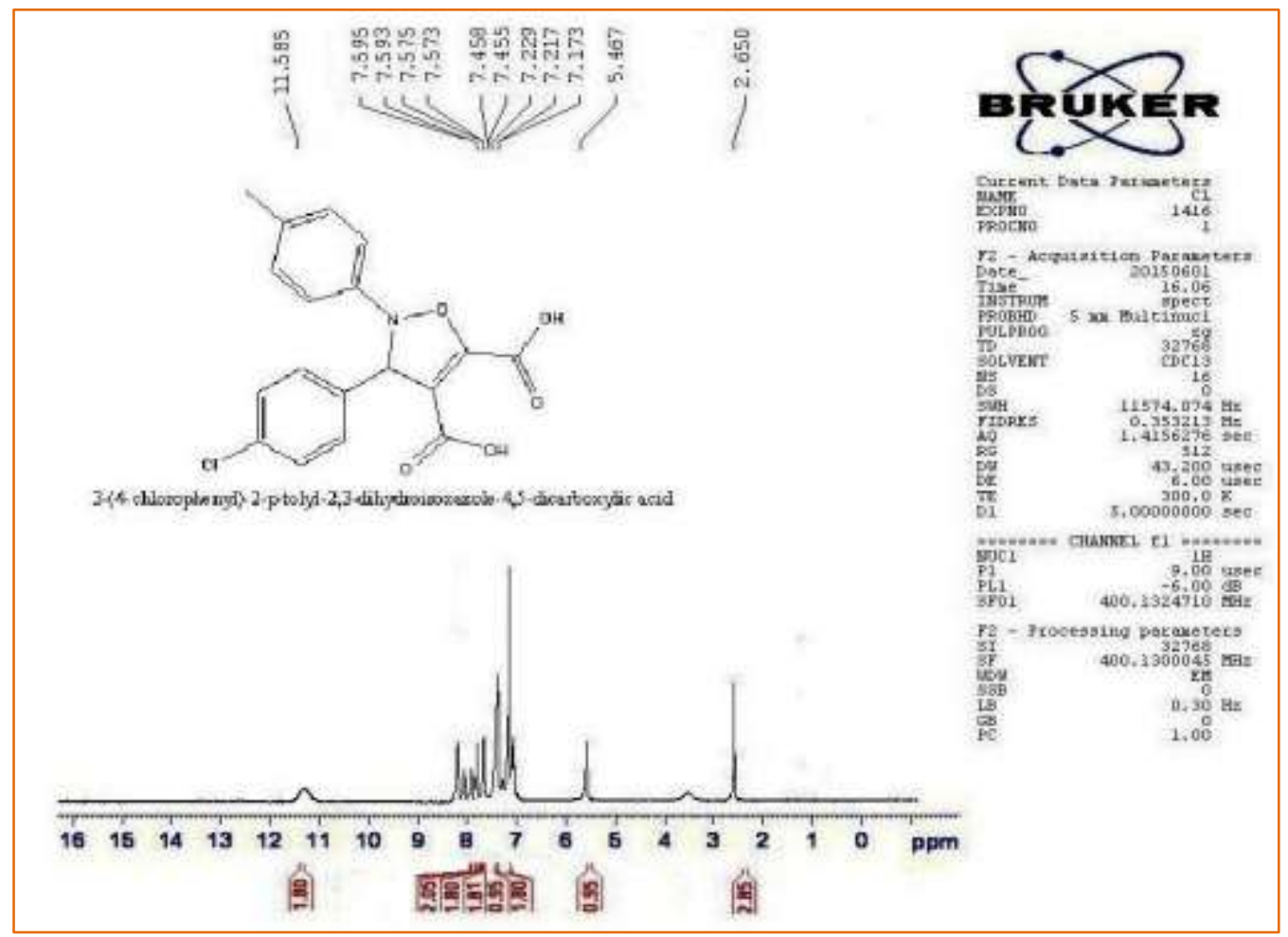

Fig (4) ${ }^{1}$ HNMR for compound (6)

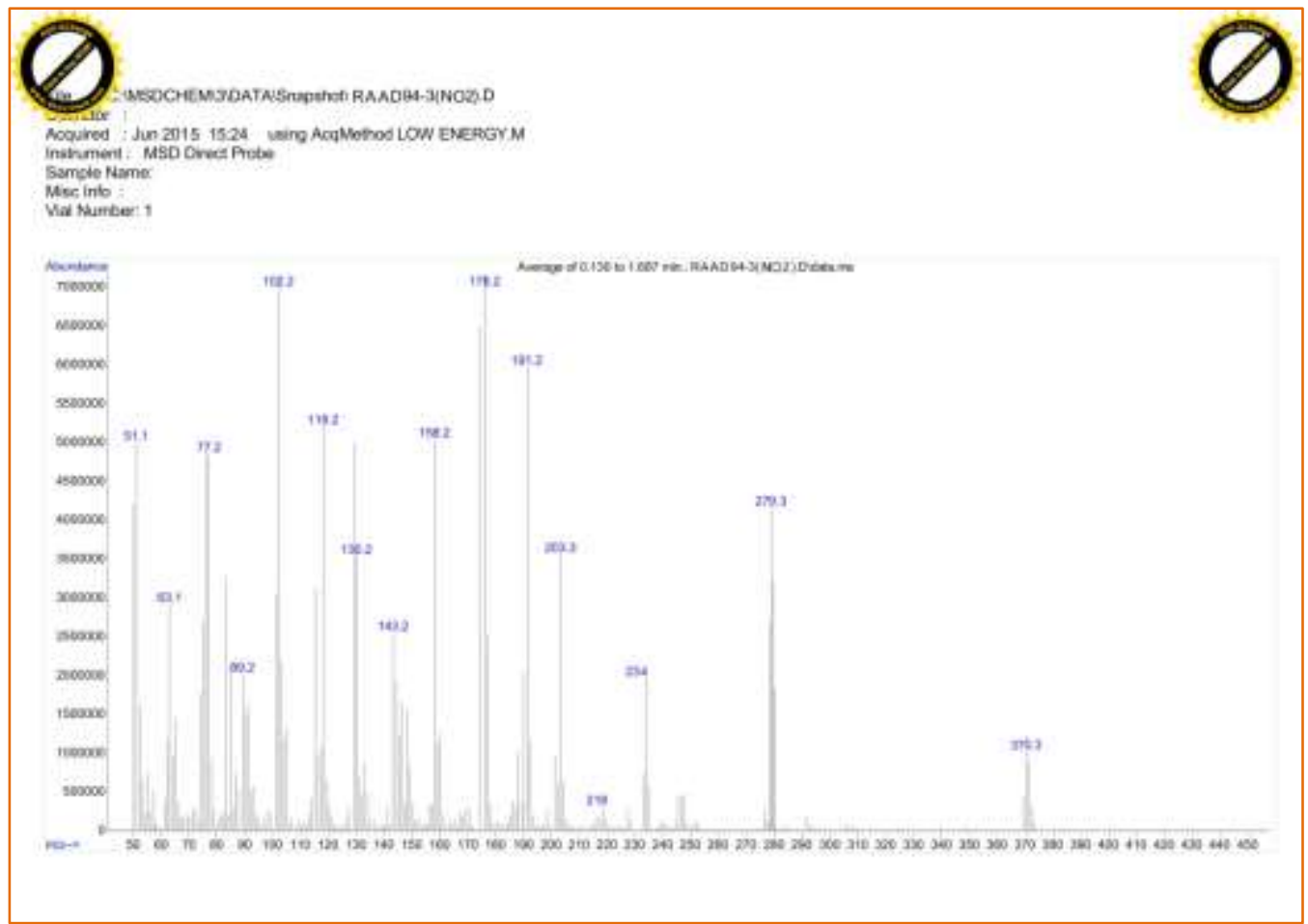

Fig (5 ) mass spectrum for compound (4) 


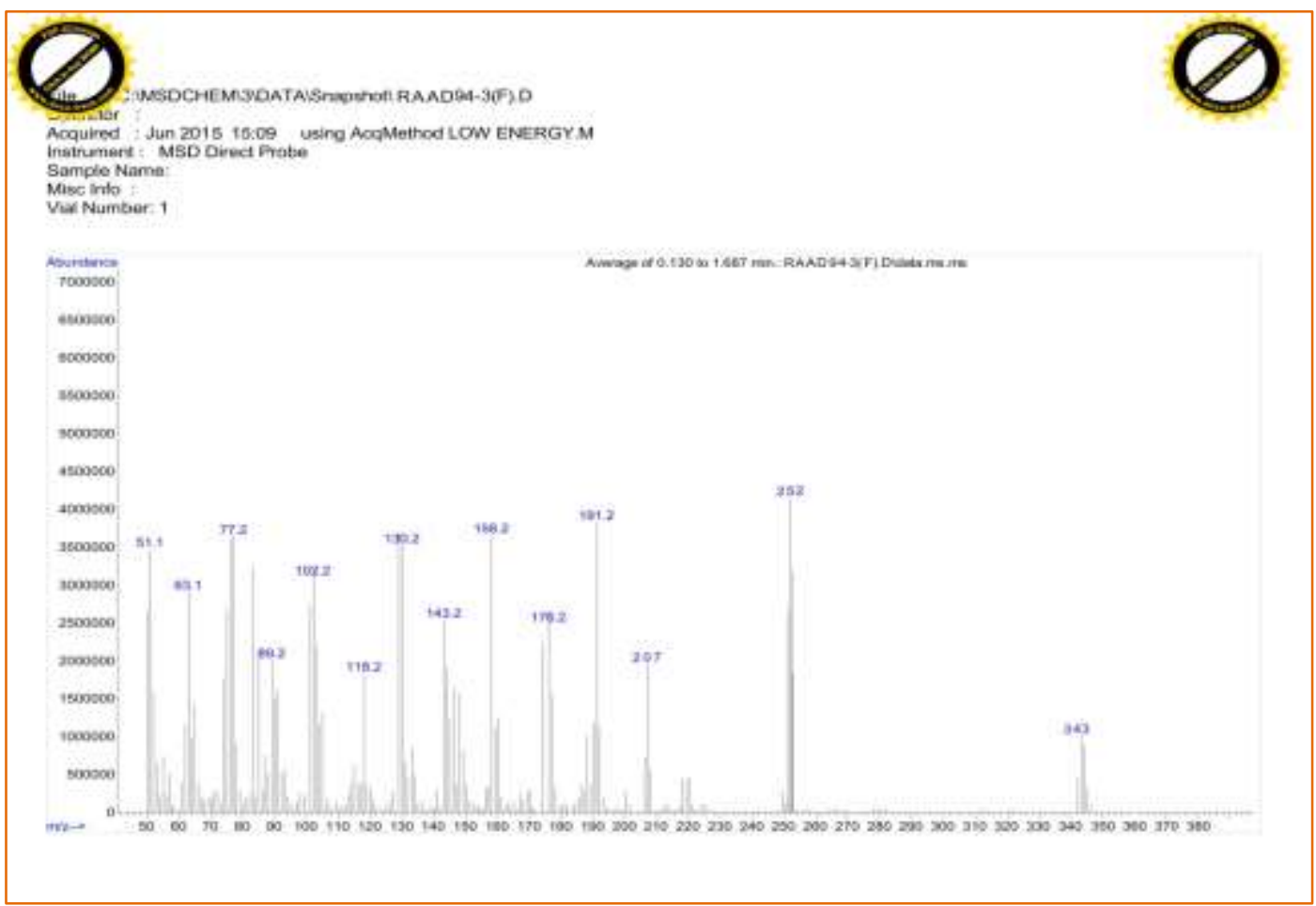

Fig (6) mass spectrum for compound (5)

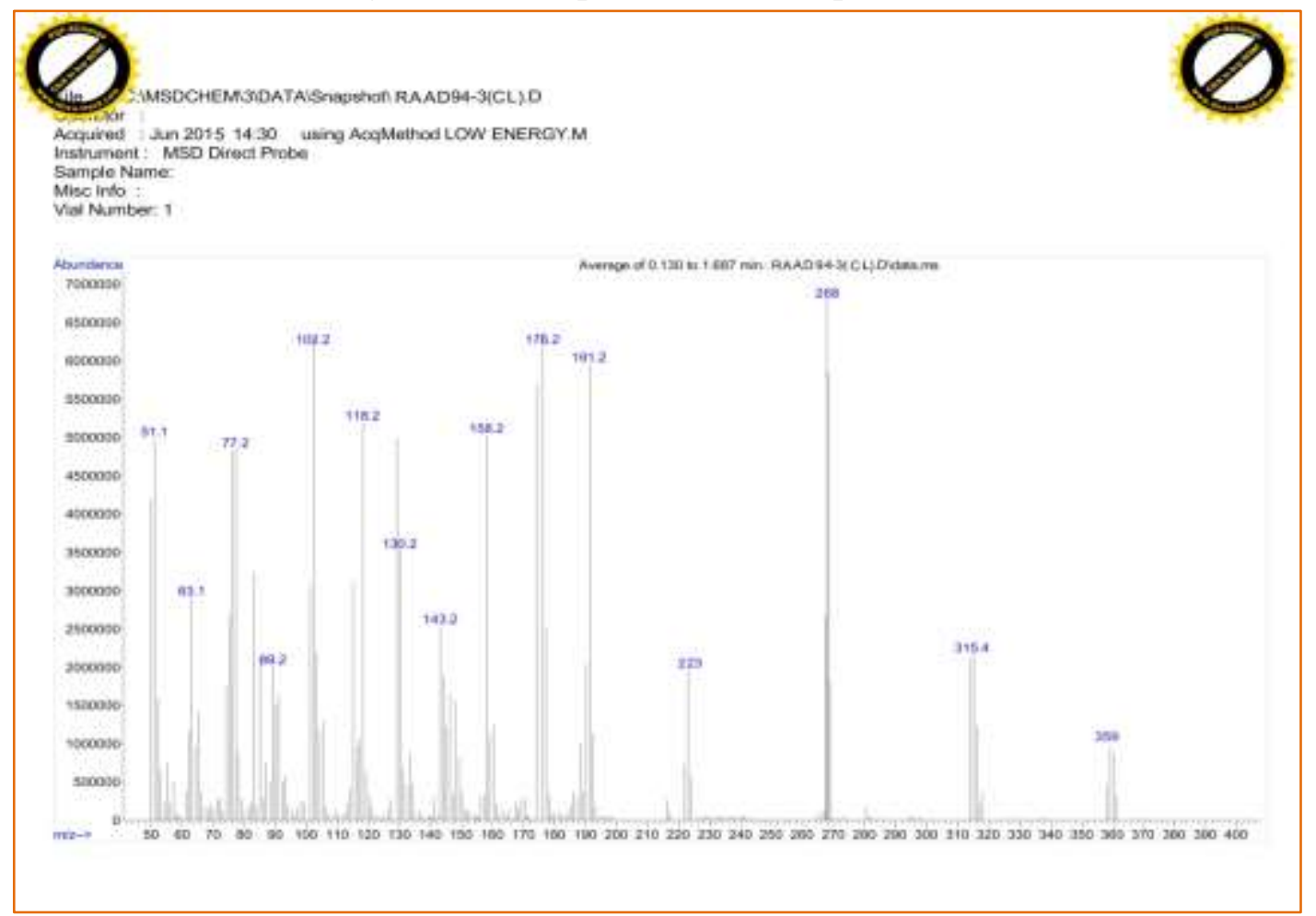

Fig (7) mass spectrum for compound (6) 
University of Thi-Qar Journal Vol.10 No.4 Dec 2015

Web Site: https://jutq.utq.edu.iq/index.php/main Email: journal@jutq.utq.edu.iq

\section{Conclusion:}

In conclusion, 1,3- dipolar cycloaddition reaction of some nitrones (1-3) with but-2ynedioic acid to give new isoxazoles (4-6).

\section{References}

1- R. Huisgen, Angew. Chem., Int. Ed. Engl., (1963) ,10,pp 565-598

2- A. Brandi, S. Cicchi, F. M. Cordero, and A. Goti ;Chem. Rev., (2014), 114 (15), pp 7317-7420

3- A. Padwa . 1,3-Dipolar Cycloaddition Chemistry Vols 1-2. Wiley Interscience, New York (1984)

4- G. Pandey, A. K. Sahoo, R.Smita , D. Trusar;J.org.chem.(1990), pp 4990-4994.

5- M.K. Werner, M.J. de los Santos, and M. Steven ;J.org.chem(1999),64, pp 4865-4873

6- G.D. Young, E. Gomez-Bengoa, and H. Amir ; J.org.chem (1999).64.pp 692-693

7- B.B. Barry , H. Lin ; Am.chem.soc (1999),121,pp 7778-7786.

8- R. Huisgen, In 1,3-Dipolar Cycloaddition Chemistry;A. Padwa, , Ed.; Wiley:

New York, (1984); Vol. 1, pp 176

9- R.S. Menon, V. Nair , Molecular Sciences and Chemical Engineering, from Comprehensive Organic Synthesis II (2 ed), (2014),4,pp 1281-1341

10- J.Malinina, T. Q. Tran, A. V. Stepakov, V. V. Gurzhiy, G. L. Starova,R. R. Kostikov, A. P. Molchanov; Tetrahedron Letters, (2014), 55 ,pp 3663-3666

11-J.Du-Ming , X. Ming-Hua ;Tetrahedron Letters （2009),50, pp2952-2955

12-M. Arnó, R.J. Zaragozá, L.R. Domingo ; Tetrahedron Asymmetry, (2004), 15,pp1541-1549

13- W.Wang, R. T. Cassell, K.S. Rein ; Tetrahedron Asymmetry, (2013), 24,pp15411549

14-M. M. Heravi, V. Zadsirjan; Tetrahedron: Asymmetry (2014),25, pp1061-1090

15-K. Grela, , L. Konopski, Tetrahedron (2010) , 66, pp3608-3613

16- M.Segi, K.Tanno, M. Kojima, M. Honda and T.Nakajima Tetrahedron Letters , (2007) ,48,pp 2303-2306

17- D. Carmona, M. P. Lamata, F. Viguri, R. Rodríguez, F. J. Lahoz;

Tetrahedron: Asymmetry 20 (2009), 20, pp1197-1205

18- K.Grela, L.Konopski ;Tetrahedron , (2010), 66,pp3614-3622

19- R.R.K.Kumar, H. M. Basappa, K. S Rangappa ;Eur.J.Med.chem.(2003),38.613

20- K.V. Gothelf and K.A. Jorgensen; Chem.Rev.(1998).98, pp 863-910

21-G. Broggini,G. Zecchi, ; Synthesis (1999),17. 905

22-J.Mulzer,organic synthesis Highlights, Verlag Chemic,Weinheim.(1991) p 77

23- K. Tadano, K. Hakuba, H. Kimura, Seiichiro Ogawa; J.org.chem.(1989),54,pp 276279

24- A.Dondoni,F.S.Merchan;synth.commun (1994).22.2200 
University of Thi-Qar Journal Vol.10 No.4 Dec 2015

Web Site: https://jutq.utq.edu.iq/index.php/main Email: journal@jutq.utq.edu.iq

25- C. L. Varela , C. Amaral , E. T. Silva ; European Journal of Medicinal Chemistry, (2014) , 87,pp 336-345

26-H.H.Salman and N.N.Majeed.J.Basrah Researches sciences (2013),39,99-111

27- J. J. Tufariello, In 1,3-Dipolar Cycloaddition Chemistry; A.Padwa, ,Ed.; J.Wiley \&S. Sons: New York, ( 1984); Vol. 2, Chapter 9.

28- K. B. Torssell, G.In Nitrile Oxides, Nitrones and Nitronates in Organic Synthesis; Feuer, H., Ed.;VCH: Weinheim, Germany, (1988).

29- R. C. F.Jones,; J. N. Martin, In Synthetic Applications of 1,3-Dipolar Cycloadditions. Chemistry Toward Heterocycles and Natural Products; Padwa, A., Pearson, W. H., Eds; John Wiley \& Sons:Hoboken, NJ, (2003); Chapter 1.

30- P. N. Confalone, E. M. Huie, Org. React. (1988), 36,1.

31- G.Tennant, In Comprehensive Organic Chemistry; Barton, D., Ollis, W. D.,Eds.; Pergamon Press: New York, ( 1979); Vol. 2, Part 8.

32- G. R.Delpierre, M. Q. Lamchen, Rev., Chem. Soc. ( 1965), 19, 329.

33- J. Hamer, A.Macaluso, Chem. Rev. (1964), 64, 473.

34- S.R. Sandler and W. Karo, Organic Functional Group Preparations, 2nd ed, Academic Press, San Diego, (1989). 3, 351-376.

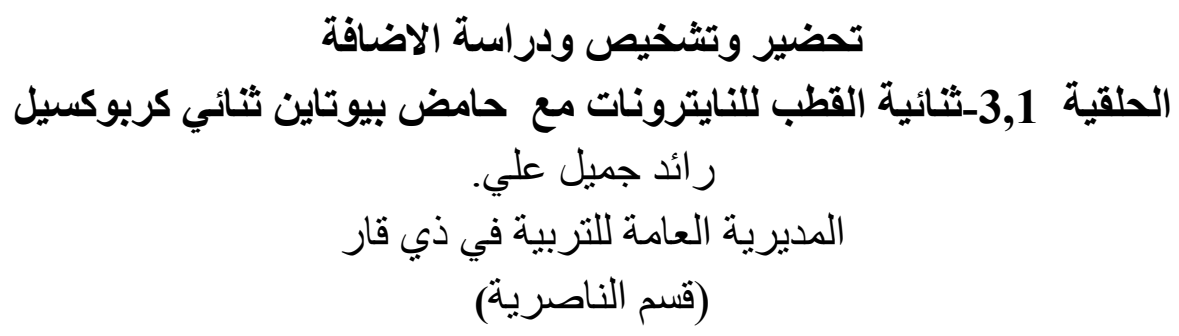

\footnotetext{
حضرت بعض مركبات النايترونات مشتقة من تفاعل نـ بار ا توليل هيدروكسيل امين مع معوضات البنز الدهايد(4-

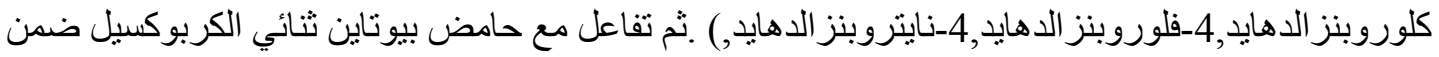

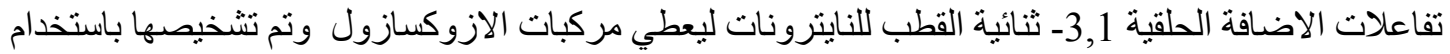
طيف الاشعة تحت الحمر اء وطيف بروتون للرنين النووي المغناطيسي وكذلك طبف الكتلة
} 
University of Thi-Qar Journal Vol.10 No.4 Dec 2015

Web Site: https://jutq.utq.edu.iq/index.php/main Email: journal@jutq.utq.edu.iq 